\title{
Ductal carcinoma in situ: a comprehensive review on current and future management for the surgeon and non-surgeon
}

\author{
Valentina V. Bonev^ \\ Breastlink Medical Group, Orange, CA, USA \\ Correspondence to: Valentina V. Bonev, MD. Breastlink Medical Group, 230 South Main Street, Suite 100, Orange, CA 92868, USA. \\ Email: valentina.bonev@breastlink.com.
}

\begin{abstract}
The management of ductal carcinoma in situ (DCIS) continues to evolve. The purpose of this review is to summarize current and future management of DCIS. DCIS comprises $20 \%$ of all newly diagnosed breast cancers in the U.S. It is diagnosed with increasing frequency due to widespread screening mammography and usually appears as a group of calcifications. Patients are frequently asymptomatic at the time of presentation. DCIS is a malignant proliferation of epithelial cells confined to the basement membrane of the breast duct and, by definition, does not metastasize. It is, however, a pre-malignant lesion with the potential for upstaging to invasive carcinoma and is managed similarly with multimodal therapy consisting of wide excision, radiotherapy (RT), and endocrine therapy (ET). DCIS has the potential for over treatment due to potentially low risk of upstaging and low mortality rates. Several large clinical trials are investigating if management can be deescalated by identifying patients with low-risk DCIS who can be safely managed with active surveillance by mammography. Furthermore, neoadjuvant endocrine therapy, anti-human epidermal growth factor receptor 2 (HER2) therapy, and immunotherapy are currently being investigated as well. A literature review of consensus guidelines and clinical trials evaluating the management of DCIS was performed. This is not a complete systematic review, but a comprehensive review on the subject. The management of DCIS is evolving and the future of care will become more tailored individually for patients.
\end{abstract}

Keywords: Endocrine therapy (ET); ductal carcinoma in situ (DCIS); breast conserving therapy (BCT); immunotherapy

Received: 27 April 2021; Accepted: 23 July 2021; Published: 30 December 2021.

doi: 10.21037/asj-21-20

View this article at: https://dx.doi.org/10.21037/asj-21-20

\section{Introduction}

Ductal carcinoma in situ (DCIS) comprises $20 \%$ of all newly diagnosed breast cancers in the U.S. $(1,2)$. The annual incidence of DCIS in the U.S. is estimated at 60,290 women (3). It is diagnosed with increasing frequency due to widespread screening mammography and approximately one in every 1,300 mammograms leads to a DCIS diagnosis (4). It usually appears as a group of calcifications on mammogram, in which a core needle biopsy is recommended. Patients are classically asymptomatic without a palpable mass, but patients who do present with a palpable mass are more likely to progress to invasive disease (5). Younger patients are more likely to present with a palpable mass and a greater extent of disease.

DCIS is a malignant proliferation of epithelial cells confined to the basement membrane of the breast duct and, by definition, does not metastasize (6). It is classified as in situ disease and is staged as TisNOM0 or stage 0 (7). It is, however, a pre-malignant lesion with the potential for upstaging to invasive carcinoma. Upstage rates on

^ ORCID: 0000-0003-1583-668X. 
final pathology after wide excision of DCIS are variable and have been reported as $8-56 \%$ and thus, it is a nonobligate precursor $(8,9)$. A validated nomogram was developed by Jakub et al. to predict the risk of upstaging (10). Preoperative factors associated with upstaging are higher grade on core needle biopsy and imaging factors, including a mass lesion, larger linear dimension and multicentric disease. In addition, suspicion of microinvasion was associated with a $50 \%$ upstage rate.

The natural progression of DCIS is uncertain but risk factors have been studied on patients with unresected DCIS who developed invasive cancer (11). Maxwell et al. is one such group that studied women who were diagnosed with DCIS on needle biopsy, but did not undergo resection for a year or more after diagnosis. Eighty-nine women with DCIS diagnosed on biopsy from 1998 to 2010 were identified. The median age at diagnosis was 75 (range, 4494) years with a median follow-up of 59 [12-180] months. Twenty-nine women (33\%) developed invasive breast cancer after a median interval of 45 [12-144] months. This risk was higher in women with high grade DCIS, younger women, and lesions with calcifications as the predominant feature on mammogram. Endocrine therapy (ET) was significantly associated with a lower rate of invasive breast cancer. Active surveillance could be an option for patients with low risk factors and is currently being investigated.

The UK Sloane Project is a prospective cohort study of 9,938 women diagnosed with screen-detected DCIS from 2003 to 2012 (12). Seventy percent of women were treated with breast conserving therapy (BCT) and 30\% with mastectomy. Of the patients who underwent radiotherapy (RT) after lumpectomy, there was a $3.1 \%$ absolute reduction in ipsilateral recurrent DCIS or invasive breast cancer. ET was prescribed to more patients after lumpectomy (14\%) than mastectomy $(8 \%)(\mathrm{P}<0.001)$. Adjuvant ET was associated with a reduction in ipsilateral recurrence whether RT was received [hazard ratio (HR) 0.57 ; $95 \%$ confidence interval (CI): 0.41-0.80] or not (HR 0.68; 95\% CI: 0.510.91 ) after lumpectomy.

Narod et al. reviewed Surveillance, Epidemiology, and End Results (SEER) data from 1988 to 2011 on 108,196 women diagnosed with DCIS (13). The mean age of diagnosis was 53.8 years old. The risk of ipsilateral invasive recurrence at 20 years was $5.9 \%$ and the risk of contralateral invasive recurrence was $6.2 \%$. At 20 years, the breast cancer-specific mortality was $3.3 \%$ and was higher for women diagnosed before 35 years old and for blacks.
RT after a lumpectomy was associated with a reduction in the risk of ipsilateral invasive recurrence at 10 years $(2.5 \%$ vs. $4.9 \%)$ but not of breast cancer-specific mortality at 10 years $(0.8 \%$ vs. $0.9 \%)$.

DCIS is similar to invasive breast cancer in that it is heterogeneous in its biology and outcomes (14). Its clinical behavior is not entirely clear, which can make management challenging and potentially leads to over treatment. The purpose of this review is to summarize current and future management of DCIS.

\section{Current management}

The National Comprehensive Cancer Network's (NCCN) workup for patients with DCIS include a history and physical exam, bilateral diagnostic mammogram, and pathology review of biopsy, including estrogen receptor status (15). Genetic testing is considered for patients who are determined to be at risk for hereditary breast cancer. Breast MRI is indicated, if appropriate. MRI can be used for staging and is more sensitive and accurate compared to mammography in detecting extent of disease, particularly for multifocal or multicentric disease (16).

A meta-analysis of nine retrospective studies on the effect of pre-operative MRI on the surgical management of DCIS found that MRI significantly increased the odds of having an initial mastectomy [odds ratio (OR) 1.72, $\mathrm{P}=0.012$ ] (17). There was no significant difference in the rate of positive margins or re-excision in women who underwent wide excision as their initial surgery. The meta-analysis concluded that pre-operative MRI did not improve surgical outcomes in women with DCIS.

A non-randomized clinical trial found that in 339 women with DCIS who underwent pre-operative MRI, 19\% of patients eligible for wide excision converted to mastectomy (18). Conversions were based on MRI findings (38\%), patient preference (38.5\%), positive margins (15.4\%), genetic test results with a mutation in a breast cancer associated gene (4.6\%), and contraindication to RT (3.1\%). A disadvantage of MRI is that it was associated with additional biopsies in $19.8 \%$ of patients. The clinical trial concluded that MRI can be useful in terms of planning management for DCIS patients.

Despite the low risk of death, DCIS is managed similarly to invasive carcinoma due to the risk of upstaging. Management is multimodal therapy that includes surgical resection, RT, and ET (19) (Figure 1). 


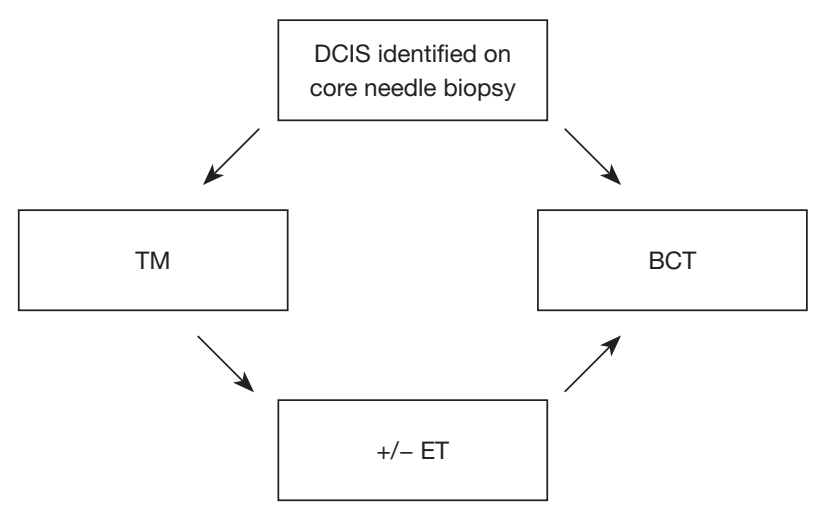

Figure 1 Current standard of care is multimodal management of DCIS. Patients can receive BCT, which consists of wide excision and radiation, or TM. Both can be followed by ET if patients are hormone receptor positive. DCIS, ductal carcinoma in situ; TM, total mastectomy; BCT, breast conserving therapy; ET, endocrine therapy.

\section{Surgical management}

Once a patient has been determined a candidate for surgery, management options include BCT or mastectomy. BCT includes a wide excision, which may also be referred to as a lumpectomy or partial mastectomy, that is typically followed by RT. The observational study by Narod et al. showed that the 10-year breast cancer specific mortality was similar when comparing mastectomy with BCT. Although, Sagara $e t a l$. analyzed SEER data and found no breast cancer specific survival benefit of surgery with low grade disease compared to patients managed without surgery (20). There was, however, a survival benefit of surgery in patients with intermediate or high-grade DCIS. The utility of surgery versus active surveillance for DCIS will be discussed in the future management section.

Approximately $70-80 \%$ of DCIS patients can be managed with wide excision (21). Candidates for wide excision are those who can achieve an oncological resection in an acceptable cosmetic manner (22). A margin of at least $2 \mathrm{~mm}$ between the DCIS and ink is recommended in a consensus statement endorsed by the American Society of Clinical Oncology, Society of Surgical Oncology, and American Society for Radiation Oncology (ASTRO) (23). The multidisciplinary panel based the consensus on a metaanalysis of 20 studies including 7,883 patients. Negative margins halved the risk of ipsilateral breast tumor recurrence (IBTR) when compared to positive margins. A $2 \mathrm{~mm}$ margin decreased the risk of IBTR when compared to smaller negative margins, but wider margins did not significantly decrease IBTR when compared to $2 \mathrm{~mm}$ margins. If a $2 \mathrm{~mm}$ margin is not achieved, a re-excision of margins should be considered.

A mastectomy has similar indications for DCIS as it does for invasive breast carcinoma. It is indicated in multicentric disease, inability to achieve negative margins, and patient preference. RT and mammography are not routinely indicated after mastectomy; therefore, some patients choose mastectomy to avoid this. Patients who have a mastectomy also have the option of having reconstruction, which can include a foreign body (tissue expanders or implants), with or without autologous reconstruction with a flap.

A cohort study from 1997 to 2006 surveyed 1,537 women with DCIS about satisfaction with their surgical and reconstruction decisions (24). Over $90 \%$ of women were reportedly satisfied with their surgery regardless of the type. Women who underwent mastectomy with reconstruction were more likely to report lower levels of satisfaction than women undergoing BCT (OR 2.98, $\mathrm{P}<0.01$ ), but over $80 \%$ of women who underwent mastectomy with reconstruction reported satisfaction with their surgical decision. Women who underwent mastectomy without reconstruction had the highest levels of satisfaction while women with implants were more likely to be dissatisfied. The study concluded that most women were satisfied with their surgical decision.

A sentinel lymph node biopsy (SLNB) is not indicated when performing wide excision for DCIS as it is, by definition, confined to the basement membrane. The NCCN, however, recommends a SLNB when a mastectomy is performed. This is due to the difficulty of performing a SLNB in a patient who already had a mastectomy due to disrupted lymphatic channels, but this is being challenged by studies showing that SLNB is still feasible with accurate detection rates $(25,26)$. Of note, when DCIS is upstaged to invasive disease on final pathology, the rate of a positive SLNB has been reported as 4-15\% (27).

\section{$R T$}

$\mathrm{RT}$ is part of the standard management for BCT. This was first demonstrated by the National Surgical Breast and Bowel Project (NSABP) B-17 trial (28). Eight hundred and thirteen patients with DCIS were randomized to wide excision with or without RT. The incidence of ipsilateral DCIS recurrence was $9 \%$ with RT versus $15.4 \%$ without RT. The incidence of invasive carcinoma recurrence was $10.7 \%$ with RT versus $19.6 \%$ without RT after 15 -year 
follow up. The addition of RT does not affect overall survival (29). Although, Sagara et al. found that when using propensity score analysis of SEER data from 1988 to 2007 , there was $0.3 \%$ survival benefit, albeit small, but statistically significant, in women who received RT (30). This significant improvement in survival was only seen in women who were higher grade, younger and had a larger tumor size.

Patients receive whole breast radiation 5 days a week for approximately 3 to 5 weeks. Patients receive a dose of $45-50.4$ Gy in 25 to 28 fractions or a hypofractionated dose of 40-42.5 Gy in 15 to 16 fractions. Patients deemed at higher risk for recurrence will also receive an additional boost of RT. The most common side effect of RT is acute skin toxicity that can occur during RT and weeks after finishing treatment (31). It can range from a mild sunburn to desquamating skin. The majority of patients have a mild skin reaction that goes away after a few weeks. Patients receiving surgery and RT are more likely to experience breast pain $(10 \%)$ when compared to patients who did not receive RT (2\%), but this pain did not significantly affect daily life activities (32). Cardiotoxicity is another concern, but the mean heart dose from RT is minimized when patients perform deep inspiration breath holding techniques during RT (33). Lastly, radiation-associated sarcoma to the breast is a rare complication that can occur years after treatment.

There are several randomized trials demonstrating the benefit of RT for DCIS. The SweDCIS trial recruited 1,067 patients with DCIS from 1987 to 1999 who underwent BCT (34). The absolute risk reduction for an ipsilateral event after RT was $12 \%$ after 20 years. The absolute risk reduction was $10 \%$ for in situ and $2.0 \%$ for invasive cancer events. The effect of RT in reducing ipsilateral events was mainly seen during the first 10 years of follow up. The Early Breast Cancer Trialists Collaborative Group (EBCTCG) compared data from four randomized trials that began on adjuvant RT versus no RT in 3,729 women with DCIS undergoing wide excision (29). RT reduced the absolute 10-year risk of any ipsilateral breast event (recurrent DCIS or invasive cancer) by $15.2 \%$ that was effective regardless of age at diagnosis, use of tamoxifen, margin status, grade, or tumor size. There was no significant effect on breast cancer mortality or allcause mortality after 10 years of follow up. The phase III trial by the Radiation Therapy Oncology Group (RTOG 9804) randomized patients with low or intermediate grade DCIS less than $2.5 \mathrm{~cm}$ in size with a minimum of $3 \mathrm{~mm}$ margins to RT versus observation after wide excision (35). The use of tamoxifen was optional. The trial was stopped early due to low accrual, but after a median follow-up of 7 years, the local failure rate was $0.9 \%$ in the RT arm versus $6.7 \%$ in the observation arm. There were no differences in disease free or overall survival.

Some patients, however, with low-risk disease and negative margins may be able to omit RT. The Eastern Cooperative Oncology Group (E5194) led an observational study that investigated excision without RT in women with low- to intermediate-grade versus high-grade DCIS (36). Eligible patients had less than $2.5 \mathrm{~cm}$ of low- to intermediate-grade DCIS or less than $1.0 \mathrm{~cm}$ of high-grade DCIS. Margins $\geq 3$ mm were required, which is greater than the current standard. Tamoxifen following excision was allowed, but not mandated. The five-year local recurrence rates for low- or intermediategrade DCIS was $6.1 \%$ versus $15.3 \%$ for high grade DCIS. These results suggest that patients with low- to intermediategrade DCIS may be better candidates for local excision without RT than those with high-grade lesions. Wong et al. also studied whether wide excision alone (without RT or tamoxifen) with margins $1 \mathrm{~cm}$ or greater is sufficient treatment for low or intermediate grade DCIS (37). In this phase II single arm prospective trial, the 10-year estimated cumulative incidence of local recurrence as $15.6 \%$. Of the local recurrences, $68 \%$ were DCIS and $32 \%$ were invasive disease while $74 \%$ of the recurrences occurred in the original quadrant. Wong et al. is currently investigating the role of wide excision in patients with low-risk DCIS with the omission of RT (NCT02926911).

The Oncotype DX Breast DCIS score assay is the first multigene assay that provides individualized estimates of 10-year risk of any local recurrence (DCIS or invasive) (38). The score ranges from 0 to 100 and is based on the expression of 12 genes that stratifies patients into risk groups. The assay includes seven cancer-related genes, which are Ki67, STK15, survivin, CCNB1, MYBL2, PR, and GSTM1, and five reference genes: ACTB, GAPDH, RPLPO, GUS, and TFRC. The three risk categories are based on score: low risk (DCIS score less than 39), intermediate risk (DCIS score $=39-54$ ), and high risk (DCIS Score $\geq 55$ ). One study assessed how the score impacted recommendations made by surgeons and radiation oncologists regarding RT following wide excision in patients with DCIS (39). The pre-score RT recommendation rate was over $70 \%$, but the score changed RT recommendations $26.4 \%$ of the time. Physicians cited the score as the most important factor in determining RT recommendations, which supports the clinical utility of the score.

DCISionRT is a score, which has been cross-validated, that was developed to provide individualized recurrence 
risk and predict the benefit of RT for DCIS patients having BCT (40). Molecular and clinical factors were used to calculate a score. Patients with a score of $\leq 3$ were classified as low risk and those with a score of greater than 3 were elevated risk. In terms of patients who were not treated with RT, the low-risk group had a 10 -year risk of invasive breast cancer at $4 \%$ while the high-risk group risk was $15 \%$. When evaluating patients who received RT, the elevated risk group received a significant benefit with a HR of 0.3 for risk of invasive breast cancer. Thus, DCISionRT can be used as a prognostic score for risk of breast cancer and predicting RT benefit.

\section{Medical therapy}

The goal of medical therapy is to reduce the risk of ipsilateral recurrence and to decrease the likelihood of a new primary in either breast. Patients may receive ET if they are estrogen receptor/progesterone receptor (ER/PR) positive and is given for 5 years post-operatively. $60-75 \%$ of DCIS lesions express estrogen and/or progesterone receptors (41). Pre-menopausal patients receive tamoxifen while post-menopausal patients may receive tamoxifen or an aromatase inhibitor. The standard dose of tamoxifen is $20 \mathrm{mg}$ daily. Patients may be offered a lower dose if the higher dose causes substantial toxicities. Side effects include hot flashes, increased risk of thromboembolic events, endometrial cancer, weight gain, and sexual dysfunction (42).

The NSABP B-24 trial randomized DCIS patients undergoing BCT to receive tamoxifen or placebo (43). Estrogen receptor status was not tested, though. Tamoxifen was found to be effective in reducing the risk of ipsilateral and contralateral invasive cancer and DCIS at 5 years. Fifteen years follow up showed that the rate of ipsilateral breast events was $16.6 \%$ in the placebo group and $13.2 \%$ in the tamoxifen group and the rate of contralateral breast events was $8.1 \%$ and $4.9 \%$, respectively.

The NSABP B-35 trial randomized over 3,100 postmenopausal women with hormone receptor positive DCIS who were undergoing BCT to 5 years of treatment with tamoxifen or anastrozole (44). When compared to tamoxifen at a median follow up of 9 years, anastrozole resulted in a lower incidence of breast cancer events but no significant difference in overall survival. The International Breast Cancer Intervention Study-II DCIS, a randomized double-blind trial from 2003 to 2012, also compared anastrozole to tamoxifen in 2,980 postmenopausal women with hormone receptor positive DCIS who underwent BCT (45). No significant difference in recurrence, including recurrent DCIS and new contralateral tumors, was found.

\section{Anti-HER2 therapy}

Currently, human epidermal growth factor receptor 2 (HER2) directed therapy is not indicated in the management of DCIS. Sagara et al. used the American College of Surgeons National Cancer Database from 2004 to 2013 to study trends in adjuvant RT and ET following BCT in women with DCIS (46). There was a trend towards decreasing use of RT, but increasing use of ET with or without RT in women who were hormone receptor positive. On the other hand, there was a trend towards increasing use of RT in women who were hormone receptor negative. The study identified a low-risk cohort, which was women over 60 years old with low grade hormone receptor positive DCIS lesions measuring less than $16 \mathrm{~mm}$ that were excised to negative margins. This cohort had a decreased use of RT, which suggests that there are select patients with DCIS who can have deescalated treatment.

\section{Future management}

\section{Surgical management}

DCIS has the potential for over treatment due to its potentially low risk of upstage and mortality rates. Current clinical trials are investigating which subgroups of DCIS patients can potentially avoid multimodal therapy and have deescalated treatment or be managed with surveillance (Table 1). The Comparison of Operative versus Monitoring and Endocrine Therapy (COMET) is a phase III randomized controlled clinical trial for low-risk DCIS $(47,48)$. Women with hormone receptor positive grade 1 or 2 DCIS are randomized to excision with or without RT and ET or to active surveillance with semi-annual mammograms and ET for 5 years. Approximately 500 patients have been accrued. The study endpoint is to compare the rate of ipsilateral invasive cancer after 2 years of follow up between the two groups.

There are two additional phase III clinical trials that are currently recruiting women in Europe: the trial of surgery versus active monitoring for low risk DCIS (LORIS) run by the University of Birmingham in the UK and the trial of management of low risk DCIS (LORD) run by the Netherlands Cancer Institute (49,50). Women are assigned to current standard of care versus active surveillance with 
Table 1 Summary of three current clinical trials that are evaluating standard therapy versus active surveillance

\begin{tabular}{llll}
\hline Clinical parameters & COMET & LORIS & LORD \\
\hline Study initiation year & 2017 & 2014 & 2017 \\
Age (years) & $\geq 40$ & $\geq 46$ & $\geq 45$ \\
Hormone receptor status & Positive & Any & Any \\
Grade & 1 and 2 & Non-high grade & 1 \\
Active surveillance & Semi-annual mammogram & Annual mammogram & Annual mammogram \\
Endpoint & Ipsilateral invasive cancer over & Ipsilateral invasive cancer over & Ipsilateral invasive cancer or \\
& 2 years & 10 years & grade 2-3 DCIS over 10 years \\
Estimated primary completed year & 2023 & 2020 & 2029 \\
\hline
\end{tabular}

COMET, Comparison of Operative versus Monitoring and Endocrine Therapy; LORIS, trial of surgery versus active monitoring for low risk DCIS; LORD, trial of management of low risk DCIS; DCIS, ductal carcinoma in situ.

annual mammogram. Hormone receptor status is not specified. The primary endpoint of the LORIS trial is the rate of ipsilateral invasive cancer over 10 years and for the LORD trial is ipsilateral invasive cancer or grade 2 to 3 DCIS over 10 years. These trials hypothesize that there is a subgroup of low-risk DCIS patients that can be safely managed with active surveillance.

\section{$R T$}

Minimizing treatment toxicity and cost is an important topic of research. Intraoperative RT (IORT) is one example of this as a single dose of radiation is delivered to the tumor bed at the time of excision in the operating room for patients who meet criteria for BCT (51). A study of 201 patients with DCIS or early-stage breast cancer analyzed initial outcomes with IORT who were treated between 2011 and 2019 (52). Patients received 20 Gy in one fraction at the time of surgery. Only four patients (2\%) had DCIS while $92.5 \%$ of patients had Stage I disease. All patients were ER positive and most patients were PR positive and HER2 negative. Median follow up was 23 months (range, $0-73$ months) with a rate of local recurrence at $2.0 \%$ and distant metastatic rate at $0.5 \%$. The study concluded that IORT can result in low rates of recurrence in low-risk patients. A prospective non-randomized trial analyzed 35 patients with pure DCIS up to $4 \mathrm{~cm}$ in maximum diameter who underwent IORT (53). At median follow-up of 36 months (range, 2-83 months), the local recurrence rate was $5.7 \%$. Five patients $(14.3 \%$ ) had positive margins requiring re-excision or mastectomy due to extensive disease. Thus, $91.4 \%$ of patients were successfully managed with lumpectomy and IORT alone.

A consensus statement from ASTRO, however, advises that patients be counseled on the higher risk of IBTR with IORT when compared to whole breast radiation (54). The statement also recommends that IORT be investigated in prospective studies.

The role of IORT in patients with DCIS is currently being studied in a clinical trial run by Columbia University (NCT03216421). The primary endpoint is rate of IBTR. The investigators propose decreased toxicity to tissue and surrounding organs, decreased healthcare costs, and improved quality of life. The investigators hypothesize that IORT is a safe alternative to whole breast irradiation. The study started in 2017 and is estimated to be completed by the end of 2025.

\section{Medical therapy}

Adjuvant ET has been shown to decrease the risk of non-invasive and invasive recurrence in DCIS patients. Currently, the role of neoadjuvant ET is being investigated. It is hypothesized that ET slows or halts disease progression and can increase the number of patients who can undergo BCT or even avoid surgery and RT.

A phase II clinical trial studied the MRI volume detected changes in post-menopausal DCIS patients who are ER positive and receiving 6 months of neoadjuvant daily oral letrozole (55). There was a $33 \%$ decrease in DCIS volume detected on MRI after 3 months of letrozole, which did not increase after 6 months of therapy. In the final surgical specimens, $8 \%$ of patients upstaged to invasive cancer and $13 \%$ of patients had no residual DCIS. Although this trial 
was single arm, it suggests that neoadjuvant ET results in decreased disease burden and can help guide future trials.

An ongoing phase II clinical trial is studying the effects of DCIS patients taking conjugated estrogens/bazedoxifene (Duavee), which is approved by the U.S. Food and Drug Administration for postmenopausal women to treat symptoms, such as hot flashes (NCT02694809). The trial has an estimated enrollment of 160 women, who will take the medication orally for 3-5 weeks before surgery. The trial will look at the changes in the breast tissue proliferation markers and if it causes any side effects. The investigators hypothesize that the drug is safe in women with DCIS patients and can reduce the risk of developing invasive carcinoma.

Tamoxifen is not without side effects and some women want to avoid taking an oral medication. There is a gel formulation of the active tamoxifen metabolite 4-hydroxytamoxifen (4-OHT) that can be applied in a transdermal fashion to the breast (56). A randomized double-blind placebo-controlled phase II trial studied this topical form against oral tamoxifen in 26 women with ER positive DCIS (57). Patients received the medication 610 weeks prior to surgery. The trial concluded that 4-OHT reached sufficient concentrations in the breast to have a biological effect but achieved lower plasma levels than oral tamoxifen. Therefore, 4-OHT is potentially as effective as oral tamoxifen but with less toxicity. These results are being studied in an ongoing phase II clinical trial with an estimated enrollment of 100 women with ER positive DCIS. Patients will receive oral versus topical form of tamoxifen for 6-10 weeks before surgery (NCT02993159).

\section{Anti-HER2 therapy}

HER2 positive DCIS has been found to be associated with a higher risk of developing invasive cancer (58). Currently, HER2 therapy is not standard management for DCIS but is currently being investigated.

NSABP B-43 is the first prospective randomized phase III multi-center clinical trial on high risk HER2 positive DCIS patients (59). The study compared woman who received BCT with or without the addition of trastuzumab. The addition of trastuzumab did not achieve the protocol goal of $36 \%$ reduction of in-breast tumor recurrence, but did achieve a statistically non-significant reduction of $19 \%$.

HER2 vaccines are being studied due to the discovery of improved survival of women with HER2 over expression who have HER2 antibodies (60). One such vaccine is the HER2 peptide pulsed dendritic cell vaccine, which produces a strong
anti-HER2 immune response and sensitizes CD4+ and CD8+ T cells (61). HER2 positive DCIS patients received intralesional and intra-nodal injections. Eighty percent of patients had an increased immune response post-injection regardless of the delivery route. Twenty eight percent of patients had no residual DCIS in the surgical specimen compared to approximately $10 \%$ when no HER2 therapy is used.

Another vaccine that has been developed is NeuVax, which is a combination nelipepimut-S, an immunogenic peptide that is derived from the HER2 protein, and granulocyte macrophage colony-stimulating factor (GM-CSF) (62). This vaccine is being compared to patients receiving GMCSF alone in DCIS patients who are HLA positive. Patients receive three vaccinations 2 weeks apart prior to resection of DCIS. The trial is analyzing the CD8+ response, cell proliferation, apoptosis, and infiltration of the DCIS lesion with immune cells as well as vaccine safety and toxicity.

A plasmid vaccine called WOKVAC is also being studied (21). It targets antigens from three proteins that are overexpressed in pre-invasive and high-risk breast lesions that are associated with progression to invasive breast cancer: insulin like growth factor receptors 1 and 2 and HER2. The vaccine elicits a strong type 1 immune response that stimulates tumor destruction and generation of cytotoxic $\mathrm{T}$ cells that kill tumor cells.

\section{Immunotherapy}

Immune checkpoint blockage is being studied as well. A Phase I clinical trial at the University of California, San Francisco is evaluating if immunotherapy can increase intra-lesional CD8 $+\mathrm{T}$ cells of high-risk DCIS patients (NCT02872025). The study defines high risk features as young age, palpable mass, negative hormone receptor status, HER2 positive, or high grade. These patients will receive two intra-lesional injections of pembrolizumab prior to current standard management. The increase in intra-lesional CD8+ $\mathrm{T}$ cells will be compared between patients receiving and not receiving pembrolizumab. The trial will also determine the maximum tolerated dose of pembrolizumab.

\section{Conclusions}

Primary care providers and general surgeons, as well as, of course, surgeons specializing in breast care, will encounter patients diagnosed with DCIS. It usually appears as a group of calcifications on mammography, at which point core needle biopsy is recommended. Once diagnosed, the patient 


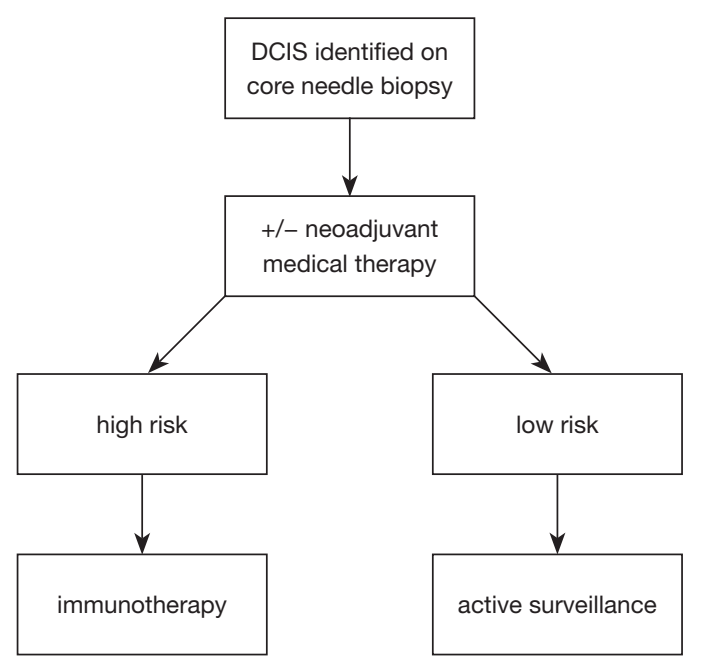

Figure 2 Potential future management of DCIS. Hormone receptor positive patients will receive neoadjuvant medical therapy, which can include endocrine and HER2 therapy. High risk DCIS patients will undergo immunotherapy and BCT while low risk patients will undergo active surveillance with mammogram. DCIS, ductal carcinoma in situ; HER2, human epidermal growth factor receptor 2; BCT, breast conserving therapy.

should be referred to a surgeon, if not already. Since it is in situ disease, it is classified as stage 0 breast cancer. Prognosis is excellent. There is a risk of upstaging to invasive disease, however, on final pathology. Currently, DCIS is managed similarly as invasive carcinoma with multimodal therapy due to this potential risk of progression. Most patients are candidates for BCT, which consists of a lumpectomy and RT. Depending on hormone receptor status, patients may also receive adjuvant ET. DCIS has the potential for over treatment due to potentially low risk of upstaging and low mortality rates. The COMET, LORIS, and LORD clinical trials are currently investigating if management can be deescalated. The goal of these trials is to identify low risk DCIS patients who can be safely managed with active surveillance by mammography. I hypothesize, that the low-risk patients will likely have low grade DCIS and be hormone receptor positive while high risk patients will have high grade DCIS and be hormone receptor negative. I predict that management of DCIS will become less aggressive (Figure 2). Neoadjuvant ET is currently being investigated and may become routinely administered in hormone receptor positive patients. Anti-HER2 therapy and immunotherapy, which are not standard of care, may shift the paradigm of DCIS management, too. As we continue to learn more about DCIS, it's management will continue to evolve and the future is promising for individualized therapy tailored to patients.

\section{Acknowledgments}

Funding: None.

\section{Footnote}

Peer Review File: Available at https://asj.amegroups.com/ article/view/10.21037/asj-21-20/prf

Conflicts of Interest: The author has completed the ICMJE uniform disclosure form (available at https://asj.amegroups. com/article/view/10.21037/asj-21-20/coif). The author has no conflicts of interest to declare.

Ethical Statement: The author is accountable for all aspects of the work in ensuring that questions related to the accuracy or integrity of any part of the work are appropriately investigated and resolved.”

Open Access Statement: This is an Open Access article distributed in accordance with the Creative Commons Attribution-NonCommercial-NoDerivs 4.0 International License (CC BY-NC-ND 4.0), which permits the noncommercial replication and distribution of the article with the strict proviso that no changes or edits are made and the original work is properly cited (including links to both the formal publication through the relevant DOI and the license). See: https://creativecommons.org/licenses/by-nc-nd/4.0/.

\section{References}

1. DeSantis CE, Ma J, Goding Sauer A, et al. Breast cancer statistics, 2017, racial disparity in mortality by state. CA Cancer J Clin 2017;67:439-48.

2. Pinder SE. Ductal carcinoma in situ (DCIS): pathological features, differential diagnosis, prognostic factors and specimen evaluation. Mod Pathol 2010;23 Suppl 2:S8-13.

3. DeSantis CE, Fedewa SA, Goding Sauer A, et al. Breast cancer statistics, 2015: Convergence of incidence rates between black and white women. CA Cancer J Clin 2016;66:31-42.

4. Ernster VL, Ballard-Barbash R, Barlow WE, et al. Detection of ductal carcinoma in situ in women undergoing screening mammography. J Natl Cancer Inst 
2002;94:1546-54.

5. Collins LC, Achacoso N, Haque R, et al. Risk factors for non-invasive and invasive local recurrence in patients with ductal carcinoma in situ. Breast Cancer Res Treat 2013;139:453-60.

6. Bane A. Ductal carcinoma in situ: what the pathologist needs to know and why. Int J Breast Cancer 2013;2013:914053.

7. Shehata M, Grimm L, Ballantyne N, et al. Ductal Carcinoma in Situ: Current Concepts in Biology, Imaging, and Treatment. J Breast Imaging 2019;1:166-76.

8. Sheaffer WW, Gray RJ, Wasif N, et al. Predictive factors of upstaging DCIS to invasive carcinoma in BCT vs mastectomy. Am J Surg 2019;217:1025-9.

9. Punglia RS, Bifolck K, Golshan M, et al. Epidemiology, Biology, Treatment, and Prevention of Ductal Carcinoma In Situ (DCIS). JNCI Cancer Spectr 2018;2:pky063.

10. Jakub JW, Murphy BL, Gonzalez AB, et al. A Validated Nomogram to Predict Upstaging of Ductal Carcinoma in Situ to Invasive Disease. Ann Surg Oncol 2017;24:2915-24.

11. Maxwell AJ, Clements K, Hilton B, et al. Risk factors for the development of invasive cancer in unresected ductal carcinoma in situ. Eur J Surg Oncol 2018;44:429-35.

12. Thompson AM, Clements K, Cheung S, et al. Management and 5-year outcomes in 9938 women with screen-detected ductal carcinoma in situ: the UK Sloane Project. Eur J Cancer 2018;101:210-9.

13. Narod SA, Iqbal J, Giannakeas V, et al. Breast Cancer Mortality After a Diagnosis of Ductal Carcinoma In Situ. JAMA Oncol 2015;1:888-96.

14. Independent UK Panel on Breast Cancer Screening. The benefits and harms of breast cancer screening: an independent review. Lancet 2012;380:1778-86.

15. National Comprehensive Cancer Network. Clinical practice guidelines in oncology: Breast Cancer. Version 5.2021. 2021. nccn.org

16. Keymeulen KBIM, Geurts SME, Lobbes MBI, et al. Population-based study of the effect of preoperative breast MRI on the surgical management of ductal carcinoma in situ. Br J Surg 2019;106:1488-94.

17. Fancellu A, Turner RM, Dixon JM, et al. Meta-analysis of the effect of preoperative breast MRI on the surgical management of ductal carcinoma in situ. Br J Surg 2015;102:883-93.

18. Lehman CD, Gatsonis C, Romanoff J, et al. Association of Magnetic Resonance Imaging and a 12-Gene Expression Assay With Breast Ductal Carcinoma In Situ Treatment.
JAMA Oncol 2019;5:1036-42.

19. Doke K, Butler S, Mitchell MP. Current Therapeutic Approaches to DCIS. J Mammary Gland Biol Neoplasia 2018;23:279-91.

20. Sagara Y, Mallory MA, Wong S, et al. Survival Benefit of Breast Surgery for Low-Grade Ductal Carcinoma In Situ: A Population-Based Cohort Study. JAMA Surg 2015;150:739-45.

21. Han MS, Khan SA. Clinical Trials for Ductal Carcinoma In Situ of the Breast. J Mammary Gland Biol Neoplasia 2018;23:293-301.

22. Nakhlis F, Morrow M. Ductal carcinoma in situ. Surg Clin North Am 2003;83:821-39.

23. Morrow M, Van Zee KJ, Solin LJ, et al. Society of Surgical Oncology-American Society for Radiation OncologyAmerican Society of Clinical Oncology Consensus Guideline on Margins for Breast-Conserving Surgery with Whole-Breast Irradiation in Ductal Carcinoma In Situ. Ann Surg Oncol 2016;23:3801-10.

24. Livingston-Rosanoff D, Trentham-Dietz A, Hampton JM, et al. Evaluation of Long-Term Satisfaction with Breast Surgery in Patients Treated for Ductal Carcinoma In Situ: A Population-Based Longitudinal Cohort Study. Ann Surg Oncol 2020;27:2628-36.

25. Karam A, Stempel M, Cody HS 3rd, et al. Reoperative sentinel lymph node biopsy after previous mastectomy. J Am Coll Surg 2008;207:543-8.

26. Intra M, Veronesi $\mathrm{P}$, Gentilini OD, et al. Sentinel lymph node biopsy is feasible even after total mastectomy. J Surg Oncol 2007;95:175-9.

27. Price A, Schnabel F, Chun J, et al. Sentinel lymph node positivity in patients undergoing mastectomies for ductal carcinoma in situ (DCIS). Breast J 2020;26:931-6.

28. Wapnir IL, Dignam JJ, Fisher B, et al. Longterm outcomes of invasive ipsilateral breast tumor recurrences after lumpectomy in NSABP B-17 and B-24 randomized clinical trials for DCIS. J Natl Cancer Inst 2011;103:478-88.

29. Early Breast Cancer Trialists' Collaborative Group (EBCTCG); Correa C, McGale P, et al. Overview of the randomized trials of radiotherapy in ductal carcinoma in situ of the breast. J Natl Cancer Inst Monogr 2010;2010:162-77.

30. Sagara Y, Freedman RA, Vaz-Luis I, et al. Patient Prognostic Score and Associations With Survival Improvement Offered by Radiotherapy After BreastConserving Surgery for Ductal Carcinoma In Situ: A Population-Based Longitudinal Cohort Study. J Clin 
Oncol 2016;34:1190-6.

31. Karlsson P. Postoperative radiotherapy after DCIS: Useful for whom? Breast 2017;34 Suppl 1:S43-6.

32. Lundstedt D, Gustafsson M, Malmström P, et al. Symptoms 10-17 years after breast cancer radiotherapy data from the randomised SWEBCG91-RT trial. Radiother Oncol 2010;97:281-7.

33. Lin A, Sharieff W, Juhasz J, et al. The benefit of deep inspiration breath hold: evaluating cardiac radiation exposure in patients after mastectomy and after breastconserving surgery. Breast Cancer 2017;24:86-91.

34. Emdin SO, Granstrand B, Ringberg A, et al. SweDCIS: Radiotherapy after sector resection for ductal carcinoma in situ of the breast. Results of a randomised trial in a population offered mammography screening. Acta Oncol 2006;45:536-43.

35. McCormick B, Winter K, Hudis C, et al. RTOG 9804: a prospective randomized trial for good-risk ductal carcinoma in situ comparing radiotherapy with observation. J Clin Oncol 2015;33:709-15.

36. Solin LJ, Gray R, Hughes LL, et al. Surgical Excision Without Radiation for Ductal Carcinoma in Situ of the Breast: 12-Year Results From the ECOG-ACRIN E5194 Study. J Clin Oncol 2015;33:3938-44.

37. Wong JS, Chen YH, Gadd MA, et al. Eight-year update of a prospective study of wide excision alone for small lowor intermediate-grade ductal carcinoma in situ (DCIS). Breast Cancer Res Treat 2014;143:343-50.

38. Solin LJ, Gray R, Baehner FL, et al. A multigene expression assay to predict local recurrence risk for ductal carcinoma in situ of the breast. J Natl Cancer Inst 2013;105:701-10.

39. Manders JB, Kuerer HM, Smith BD, et al. Clinical Utility of the 12-Gene DCIS Score Assay: Impact on Radiotherapy Recommendations for Patients with Ductal Carcinoma In Situ. Ann Surg Oncol 2017;24:660-8.

40. Bremer T, Whitworth PW, Patel R, et al. A Biological Signature for Breast Ductal Carcinoma In Situ to Predict Radiotherapy Benefit and Assess Recurrence Risk. Clin Cancer Res 2018;24:5895-901.

41. Hird RB, Chang A, Cimmino V, et al. Impact of estrogen receptor expression and other clinicopathologic features on tamoxifen use in ductal carcinoma in situ. Cancer 2006;106:2113-8.

42. Heery M, Corbett P, Zelkowitz R. Precautions for Patients Taking Tamoxifen. J Adv Pract Oncol 2018;9:78-83.

43. Fisher B, Dignam J, Wolmark N, et al. Tamoxifen in treatment of intraductal breast cancer: National Surgical
Adjuvant Breast and Bowel Project B-24 randomised controlled trial. Lancet 1999;353:1993-2000.

44. Margolese RG, Cecchini RS, Julian TB, et al. Anastrozole versus tamoxifen in postmenopausal women with ductal carcinoma in situ undergoing lumpectomy plus radiotherapy (NSABP B-35): a randomised, double-blind, phase 3 clinical trial. Lancet 2016;387:849-56.

45. Forbes JF, Sestak I, Howell A, et al. Anastrozole versus tamoxifen for the prevention of locoregional and contralateral breast cancer in postmenopausal women with locally excised ductal carcinoma in situ (IBIS-II DCIS): a double-blind, randomised controlled trial. Lancet 2016;387:866-73.

46. Sagara Y, Freedman RA, Wong SM, et al. Trends in adjuvant therapies after breast-conserving surgery for hormone receptor-positive ductal carcinoma in situ: findings from the National Cancer Database, 2004-2013. Breast Cancer Res Treat 2017;166:583-92.

47. Hwang ES, Hyslop T, Lynch T, et al. The COMET (Comparison of Operative versus Monitoring and Endocrine Therapy) trial: a phase III randomised controlled clinical trial for low-risk ductal carcinoma in situ (DCIS). BMJ Open 2019;9:e026797.

48. Youngwirth LM, Boughey JC, Hwang ES. Surgery versus monitoring and endocrine therapy for low-risk DCIS: The COMET Trial. Bull Am Coll Surg 2017;102:62-3.

49. Francis A, Thomas J, Fallowfield L, et al. Addressing overtreatment of screen detected DCIS; the LORIS trial. Eur J Cancer 2015;51:2296-303.

50. Elshof LE, Tryfonidis K, Slaets L, et al. Feasibility of a prospective, randomised, open-label, international multicentre, phase III, non-inferiority trial to assess the safety of active surveillance for low risk ductal carcinoma in situ - The LORD study. Eur J Cancer 2015;51:1497-510.

51. Khan AJ, Arthur DW, Vicini FA. On the road to intraoperative radiotherapy: more 'proceed with caution' signs. Oncology (Williston Park) 2013;27:113-4, 122.

52. Obi E, Tom MC, Manyam BV, et al. Outcomes with intraoperative radiation therapy for early-stage breast cancer. Breast J 2020;26:454-7.

53. Rivera R, Banks A, Casillas-Lopez A, et al. Targeted Intraoperative Radiotherapy for the Management of Ductal Carcinoma In Situ of the Breast. Breast J 2016;22:63-74.

54. Correa C, Harris EE, Leonardi MC, et al. Accelerated Partial Breast Irradiation: Executive summary for the update of an ASTRO Evidence-Based Consensus 
Statement. Pract Radiat Oncol 2017;7:73-9.

55. Hwang ES, Duong S, Bedrosian I, et al. Abstract GS505: primary endocrine therapy for ER-positive ductal carcinoma in situ (DCIS) CALGB 40903 (Alliance). Cancer Res 2018;78:SABCS17-GS5-05.

56. Lee O, Ivancic D, Allu S, et al. Local transdermal therapy to the breast for breast cancer prevention and DCIS therapy: preclinical and clinical evaluation. Cancer Chemother Pharmacol 2015;76:1235-46.

57. Lee O, Page K, Ivancic D, et al. A randomized phase II presurgical trial of transdermal 4-hydroxytamoxifen gel versus oral tamoxifen in women with ductal carcinoma in situ of the breast. Clin Cancer Res 2014;20:3672-82.

58. Visser LL, Elshof LE, Schaapveld M, et al. Clinicopathological Risk Factors for an Invasive Breast Cancer Recurrence after Ductal Carcinoma In Situ-A Nested Case-Control Study. Clin Cancer Res

doi: $10.21037 /$ asj-21-20

Cite this article as: Bonev VV. Ductal carcinoma in situ: a comprehensive review on current and future management for the surgeon and non-surgeon. AME Surg J 2021;1:27.
2018;24:3593-601.

59. Siziopikou KP, Anderson SJ, Cobleigh MA, et al. Preliminary results of centralized HER2 testing in ductal carcinoma in situ (DCIS): NSABP B-43. Breast Cancer Res Treat 2013;142:415-21.

60. Tabuchi Y, Shimoda M, Kagara N, et al. Protective effect of naturally occurring anti-HER2 autoantibodies on breast cancer. Breast Cancer Res Treat 2016;157:55-63.

61. Lowenfeld L, Mick R, Datta J, et al. Dendritic Cell Vaccination Enhances Immune Responses and Induces Regression of HER2pos DCIS Independent of Route: Results of Randomized Selection Design Trial. Clin Cancer Res 2017;23:2961-71.

62. Clifton GT, Peoples GE, Mittendorf EA. The development and use of the E75 (HER2 369-377) peptide vaccine. Future Oncol 2016;12:1321-9. 